\title{
On Moment Generating Function of Generalized Order Statistics from Erlang-Truncated Exponential Distribution
}

\author{
Anamika Kulshrestha, R. U. Khan, Devendra Kumar \\ Department of Statistics and Operations Research, Aligarh Muslim University, Aligarh, India \\ Email: aruke@rediffmail.com
}

Received August 28, 2012; revised September 30, 2012; accepted October 13, 2012

\begin{abstract}
In this paper explicit expressions and some recurrence relations are derived for marginal and joint moment generating functions of generalized order statistics from Erlang-truncated exponential distribution. The results for $k$-th record values and order statistics are deduced from the relations derived. Further, a characterizing result of this distribution on using the conditional expectation of function of generalized order statistics is discussed.
\end{abstract}

Keywords: Generalized Order Statistics; Order Statistics; Record Values; Erlang-Truncated Exponential Distribution; Marginal and Joint Moment Generating Function; Recurrence Relations and Characterization

\section{Introduction}

A random variable $X$ is said to have Erlang-truncated exponential distribution if its probability density function $(p d f)$ is of the form

$$
f(x)=\theta\left(1-\mathrm{e}^{-\lambda}\right) \mathrm{e}^{-\theta x\left(1-\mathrm{e}^{-\lambda}\right)}, x \geq 0, \theta, \lambda>0
$$

and the corresponding distribution function $(d f)$ is

$$
\bar{F}(x)=\mathrm{e}^{-\theta x\left(1-\mathrm{e}^{-\lambda}\right)}, x \geq 0, \theta, \lambda>0,
$$

where

$$
\bar{F}(x)=1-F(x) .
$$

For more details on this distribution and its applications one may refer to [1].

[2] introduced and extensively studied the generalized order statistics (gos). The order statistics, sequential order statistics, Stigler's order statistics, record values are special cases of gos . Suppose $X(1, n, m, k), \cdots$, $X(n, n, m, k)$ are $n$ gos from an absolutely continuous distribution function $(d f) \quad F(x)$ with the corresponding probability density function $(p d f) f(x)$. Their joint $p d f$ is

$$
\begin{aligned}
& k\left(\prod_{j=1}^{n-1} \gamma_{j}\right)\left(\prod_{i=1}^{n-1}\left[1-F\left(x_{i}\right)\right]^{m} f\left(x_{i}\right)\right) \\
& \times\left[1-F\left(x_{n}\right)\right]^{k-1} f\left(x_{n}\right)
\end{aligned}
$$

for $F^{-1}(0+)<x_{1} \leq x_{2} \leq \cdots \leq x_{n}<F^{-1}(1), m \geq-1$, $\gamma_{r}=k+(n-r)(m+1)>0, \quad r=1,2, \cdots, n-1, k \geq 1$ and $n$ is a positive integer.
Choosing the parameters appropriately, models such as ordinary order statistics $\left(\gamma_{i}=n-i+1 ; i=1,2, \cdots, n\right.$, i.e. $\left.m_{1}=m_{2}=\cdots=m_{n-1}=0, k=1\right), k$-th record values $\left(\gamma_{i}=k\right.$, i.e. $\left.m_{1}=m_{2}=\cdots=m_{n-1}=-1, k \in N\right)$, sequential order statistics $\left(\gamma_{i}=(n-i+1) \alpha_{i} ; \alpha_{1}, \alpha_{2}, \cdots, \alpha_{n}>0\right)$, order statistics with non-integral sample size $\left(\gamma_{i}=\alpha-i+1 ; \alpha>0\right)$, Pfeifer's record values $\left(\gamma_{i}=\beta_{i} ; \beta_{1}, \beta_{2}, \cdots, \beta_{n}>0\right)$ and progressive type II censored order statistics $\left(m_{i} \in N, k \in N\right)$ are obtained [2, 3].

The marginal $p d f$ of the $r$-th gos, $X(r, n, m, k)$, $1 \leq r \leq n$, is

$$
\begin{aligned}
& f_{X(r, n, m, k)}(x) \\
& =\frac{C_{r-1}}{(r-1) !}[\bar{F}(x)]^{\gamma_{r}-1} f(x) g_{m}^{r-1}(F(x))
\end{aligned}
$$

and the joint pdf of $X(r, n, m, k)$ and $X(s, n, m, k)$, $1 \leq r<s \leq n$, is

$$
\begin{aligned}
& f_{X(r, n, m, k), X(s, n, m, k)}(x, y) \\
& =\frac{C_{s-1}}{(r-1) !(s-r-1) !}[\bar{F}(x)]^{m} g_{m}^{r-1}(F(x)) \\
& \times\left[h_{m}(F(y))-h_{m}(F(x))\right]^{s-r-1} \\
& \times[\bar{F}(y)]^{\gamma_{s}-1} f(x) f(y), \\
& \alpha \leq x<y \leq \beta,
\end{aligned}
$$

where 


$$
C_{r-1}=\prod_{i=1}^{r} \gamma_{i}, h_{m}(x)= \begin{cases}-\frac{1}{m+1}(1-x)^{m+1}, & m \neq-1 \\ -\ln (1-x), & m=-1\end{cases}
$$

and $g_{m}(x)=h_{m}(x)-h_{m}(0), x \in[0,1)$.

[4-6] have established recurrence relations for moment generating functions of record values from Pareto and Gumble, power function and extreme value distributions.

Recurrence relations for marginal and joint moment generating functions of gos from power function distribution are derived by [7]. [8,9] have established recurrence relations for conditional and joint moment generating functions of gos based on mixed population, respectively. [10] has established explicit expressions and some recurrence relations for moment generating function of gos from Gompertz distribution.

In the present study, we establish exact expressions and some recurrence relations for marginal and joint moment generating functions of gos from Erlang-truncated exponential distribution. Results for order statistics and record values are deduced as special cases and a characterization of this distribution is obtained by using the conditional expectation of function of gos.

\section{Relations for Marginal Moment Generating Functions}

Note that for Erlang-truncated exponential distribution defined in (1).

$$
f(x)=\theta\left(1-\mathrm{e}^{-\lambda}\right) \bar{F}(x) .
$$

The relation in (6) will be exploited in this paper to derive exact expressions and some recurrence relations for the moment generating functions of gos from the Erlang-truncated exponential distribution.

Let us denote the marginal moment generating functions of $X(r, n, m, k)$ by $M_{X(r, n, m, k)}(t)$ and its $j$-th derivative by $M_{X(r, n, m, k)}^{(j)}(t)$.

We shall first establish the explicit expression for $M_{X(r, n, m, k)}(t)$. Using (4) and (6), we have when $m \neq-1$

$$
M_{X(r, n, m, k)}(t)=\frac{\theta\left(1-\mathrm{e}^{-\lambda}\right) C_{r-1}}{(r-1) !} I\left(\gamma_{r}, r-1\right),(7)
$$

where

$$
I(a, b)=\int_{0}^{\infty} \mathrm{e}^{t x}[\bar{F}(x)]^{a} g_{m}^{b}(F(x)) \mathrm{d} x .
$$

On expanding $g_{m}^{b}(F(x))=\left[\frac{1}{m+1}\left\{1-(\bar{F}(x))^{m+1}\right\}\right]^{b}$ binomially in (8), we get when $m \neq-1$

$$
I(a, b)=A \int_{0}^{\infty} \mathrm{e}^{t x}[\bar{F}(x)]^{a+u(m+1)} \mathrm{d} x,
$$

where

$$
A=\frac{1}{(m+1)^{b}} \sum_{u=0}^{b}(-1)^{u}\left(\begin{array}{l}
b \\
u
\end{array}\right) .
$$

On substituting for $\bar{F}(x)$ from (2) in (9), we have

$$
\begin{aligned}
I(a, b) & =A \int_{0}^{\infty} \mathrm{e}^{-x\left[\theta\left(1-\mathrm{e}^{-\lambda}\right)\{a+u(m+1)\}-t\right]} \mathrm{d} x \\
& =\frac{A}{\left[\theta\left(1-\mathrm{e}^{-\lambda}\right)\{a+u(m+1)\}-t\right]} .
\end{aligned}
$$

Now on substituting for $I\left(\gamma_{r}, r-1\right)$ from (10) in (7) and simplifying, we obtain when $m \neq-1$

$$
\begin{aligned}
M_{X(r, n, m, k)}(t) & =\frac{C_{r-1}}{(r-1) !(m+1)^{r-1}} \sum_{u=0}^{r-1}(-1)^{u}\left(\begin{array}{c}
r-1 \\
u
\end{array}\right) \\
& \times \frac{1}{\left[\gamma_{r-u}-\left\{t / \theta\left(1-\mathrm{e}^{-\lambda}\right)\right\}\right]} .
\end{aligned}
$$

When $m=-1$, we have

$$
M_{X(r, n, m, k)}(t)=\frac{0}{0} \text { as } \sum_{u=0}^{r-1}(-1)^{u}\left(\begin{array}{c}
r-1 \\
u
\end{array}\right)=0 .
$$

Since (11) is of the form $0 / 0$ at $m=-1$, therefore, we have

$$
\begin{aligned}
& M_{X(r, n, m, k)}(t)=\frac{C_{r-1}}{(r-1) !} \sum_{u=0}^{r-1}(-1)^{u}\left(\begin{array}{c}
r-1 \\
u
\end{array}\right) \\
& \times \frac{\left[k+(n-r+u)(m+1)-\left\{t / \theta\left(1-\mathrm{e}^{-\lambda}\right)\right\}\right]^{-1}}{(m+1)^{r-1}} .
\end{aligned}
$$

Differentiating numerator and denominator of (12) $(r-1)$ times with respect to $m$, we get

$$
\begin{aligned}
& M_{X(r, n, m, k)}(t)=\frac{C_{r-1}}{(r-1) !} \sum_{u=0}^{r-1}(-1)^{u+(r-1)}\left(\begin{array}{c}
r-1 \\
u
\end{array}\right) \\
& \times \frac{(n-r+u)^{r-1}}{\left[k+(n-r+u)(m+1)-\left\{t / \theta\left(1-\mathrm{e}^{-\lambda}\right)\right\}\right]^{r}} \\
& =\frac{C_{r-1}}{(r-1) !} \sum_{u=0}^{r-1}(-1)^{u}\left(\begin{array}{c}
r-1 \\
u
\end{array}\right) \\
& \times \frac{(r-n-u)^{r-1}}{\left[k+(n-r+u)(m+1)-\left\{t / \theta\left(1-\mathrm{e}^{-\lambda}\right)\right\}\right]^{r}} .
\end{aligned}
$$

On applying L' Hospital rule, we have

$$
\begin{aligned}
& \lim _{m \rightarrow-1} M_{X(r, n, m, k)}(t)=\frac{C_{r-1}}{(r-1) !\left[k-\left\{t / \theta\left(1-\mathrm{e}^{-\lambda}\right)\right\}\right]^{r}} \\
& \times \sum_{u=0}^{r-1}(-1)^{u}\left(\begin{array}{c}
r-1 \\
u
\end{array}\right)(r-n-u)^{r-1} .
\end{aligned}
$$


But for all integers $n \geq 0$ and for all real numbers $x$, we have [11]

$$
\sum_{i=0}^{n}(-1)^{i}\left(\begin{array}{l}
n \\
i
\end{array}\right)(x-i)^{n}=n !
$$

Therefore,

$$
\sum_{u=0}^{b}(-1)^{u+b}\left(\begin{array}{l}
b \\
u
\end{array}\right) u^{b}=b !
$$

Now on substituting (14) in (13), we find that

$$
M_{X(r, n,-1, k)}(t)=M_{Y_{r}^{(k)}}(t)=\frac{1}{\left[1-\left\{t / k \theta\left(1-\mathrm{e}^{-\lambda}\right)\right\}\right]^{r}} \text {. }
$$

Differentiating $M_{X(r, n, m, k)}(t)$ with respect to $t$ and evaluating at $t=0$, we get the mean of the $r$-th gos when $m>-1$

$$
\begin{aligned}
& E[X(r, n, m, k)]=\frac{C_{r-1}}{(r-1) ! \theta\left(1-\mathrm{e}^{-\lambda}\right)(m+1)^{r-1}} \\
& \times \sum_{u=0}^{r-1}(-1)^{u}\left(\begin{array}{c}
r-1 \\
u
\end{array}\right) \frac{1}{\left(\gamma_{r-u}\right)^{2}}
\end{aligned}
$$

and when $m=-1$ that

$$
E[X(r, n,-1, k)]=E\left[Y_{r}^{(k)}\right]=\frac{r}{k \theta\left(1-\mathrm{e}^{-\lambda}\right)}
$$

as obtained by [12] for exponential distribution at $\theta\left(1-\mathrm{e}^{-\lambda}\right)=c$.

\section{Special Cases}

1) Putting $m=0, k=1$ in (11) and (17), the explicit formula for marginal moment generating function and mean of order statistics from Erlang-truncated exponential distribution can be obtained as

$$
\begin{aligned}
M_{X_{r: n}}(t) & =C_{r: n} \sum_{u=0}^{r-1}(-1)^{u}\left(\begin{array}{c}
r-1 \\
u
\end{array}\right) \\
& \times \frac{1}{\left[n-r+u+1-\left\{t / \theta\left(1-\mathrm{e}^{-\lambda}\right)\right\}\right]}
\end{aligned}
$$

and

$$
E\left[X_{r: n}\right]=\frac{C_{r: n}}{\theta\left(1-\mathrm{e}^{-\lambda}\right)} \sum_{u=0}^{r-1}(-1)^{u}\left(\begin{array}{c}
r-1 \\
u
\end{array}\right) \frac{1}{(n-r+u+1)^{2}},
$$

where

$$
C_{r: n}=\frac{n !}{(r-1) !(n-r) !} .
$$

2) Setting $k=1$ in (16) and (18), the results for upper records from Erlang-truncated exponential distribution may be obtained in the form

$$
M_{Y_{r}^{(1)}}(t)=M_{X_{U(r)}}(t)=\frac{1}{\left[1-\left\{t / \theta\left(1-\mathrm{e}^{-\lambda}\right)\right\}\right]^{r}}
$$

and

$$
E\left[Y_{r}^{(1)}\right]=E\left[X_{U(r)}\right]=\frac{r}{\theta\left(1-\mathrm{e}^{-\lambda}\right)}
$$

as obtained by [13] for exponential distribution at $\theta\left(1-\mathrm{e}^{-\lambda}\right)=c$.

A recurrence relation for marginal moment generating function for gos from $d f$ (1) can be obtained in the following theorem.

Theorem 2.1 For the distribution given in (1) and for $2 \leq r \leq n, n \geq 2, k=1,2, \cdots$,

$$
\begin{aligned}
& \left(1-\frac{t}{\theta\left(1-\mathrm{e}^{-\lambda}\right) \gamma_{r}}\right) M_{X(r, n, m, k)}^{(j)}(t) \\
& =M_{X(r-1, n, m, k)}^{(j)}(t)+\frac{j}{\theta\left(1-\mathrm{e}^{-\lambda}\right) \gamma_{r}} M_{X(r, n, m, k)}^{(j-1)}(t) .
\end{aligned}
$$

Proof [10] has shown that for a positive integer $k, 1 \leq r \leq n, n \geq 2$,

$$
\begin{aligned}
& M_{X(r, n, m, k)}(t)-M_{X(r-1, n, m, k)}(t) \\
& =\frac{t C_{r-1}}{(r-1) ! \gamma_{r}} \times \int_{\alpha}^{\beta} \mathrm{e}^{t x}[\bar{F}(x)]^{\gamma_{r}} g_{m}^{r-1}(F(x)) \mathrm{d} x .
\end{aligned}
$$

On substituting for $\bar{F}(x)$ from (6) in (20) and simplifying the resulting expression, we find that

$$
\begin{aligned}
& M_{X(r, n, m, k)}(t)-M_{X(r-1, n, m, k)}(t) \\
& =\frac{t}{\theta\left(1-\mathrm{e}^{-\lambda}\right) \gamma_{r}} M_{X(r, n, m, k)}(t) .
\end{aligned}
$$

Differentiating both the sides of (21) $j$ times with respect to $t$, we get

$$
\begin{aligned}
M_{X(r, n, m, k)}^{(j)}(t) & =M_{X(r-1, n, m, k)}^{(j)}(t) \\
& +\frac{t}{\theta\left(1-\mathrm{e}^{-\lambda}\right) \gamma_{r}} M_{X(r, n, m, k)}^{(j)}(t) \\
& +\frac{j}{\theta\left(1-\mathrm{e}^{-\lambda}\right) \gamma_{r}} M_{X(r, n, m, k)}^{(j-1)}(t) .
\end{aligned}
$$

The recurrence relation in (19) is derived simply by rewriting the above equation.

At $t=0$ in (19), we obtain the recurrence relations for moments of gos from Erlang-truncated exponential distribution in the form

$$
\begin{aligned}
& E\left[X^{j}(r, n, m, k)\right]=E\left[X^{j}(r-1, n, m, k)\right] \\
& +\frac{j}{\theta\left(1-\mathrm{e}^{-\lambda}\right) \gamma_{r}} E\left[X^{j-1}(r, n, m, k)\right] .
\end{aligned}
$$


Remark 2.1 Putting $m=0, k=1$ in (19) and (22), we can get the relations for marginal moment generating function and moments of order statistics for Erlang-truncated exponential distribution as

$$
\begin{aligned}
& \left(1-\frac{t}{\theta\left(1-\mathrm{e}^{-\lambda}\right)(n-r+1)}\right) M_{X_{r-n}}^{(j)}(t) \\
& =M_{X_{r-1 ; n}}^{(j)}(t)+\frac{j}{\theta\left(1-\mathrm{e}^{-\lambda}\right)(n-r+1)} M_{X_{r-n}}^{(j-1)}(t)
\end{aligned}
$$

and

$$
\begin{aligned}
E\left[X_{r: n}^{j}\right] & =E\left[X_{r-1: n}^{j}\right] \\
& +\frac{j}{\theta\left(1-\mathrm{e}^{-\lambda}\right)(n-r+1)} E\left[X_{r: n}^{j-1}\right] .
\end{aligned}
$$

Remark 2.2 Setting $m=-1$ and $k \geq 1$ in (19) and (22), relations for record values can be obtained as

$$
\begin{aligned}
& \left(1-\frac{t}{k \theta\left(1-\mathrm{e}^{-\lambda}\right)}\right) M_{Y_{r}^{(k)}}^{(j)}(t) \\
& =M_{Y_{r-1}^{(k)}}^{(j)}(t)+\frac{j}{k \theta\left(1-\mathrm{e}^{-\lambda}\right)} M_{Y_{r}^{(k)}}^{(j-1)}(t)
\end{aligned}
$$

and

$$
\begin{aligned}
E\left[\left(Y_{r}^{(k)}\right)^{j}\right] & =E\left[\left(Y_{(r-1)}^{(k)}\right)^{j}\right] \\
& +\frac{j}{k \theta\left(1-\mathrm{e}^{-\lambda}\right)} E\left[\left(Y_{r}^{(k)}\right)^{j-1}\right],
\end{aligned}
$$

for $k=1$

$$
\begin{aligned}
E\left[X_{U(r)}^{j}\right] & =E\left[X_{U(r-1)}^{j}\right] \\
& +\frac{j}{\theta\left(1-\mathrm{e}^{-\lambda}\right)} E\left[X_{U(r)}^{j-1}\right] .
\end{aligned}
$$

Remark 2.3 At $j=1, \theta\left(1-\mathrm{e}^{-\lambda}\right)=c$ in (22), the result for single moments of gos obtained by [2] for exponential distribution is deduced.

\section{Relations for Joint Moment Generating Functions}

Before coming to the main results we shall prove the following Lemmas.

Lemma 3.1 For the Erlang-truncated exponential distribution as given in (1) and non-negative integers $a, b$ and $c$ with $m \neq-1$,

$$
\begin{aligned}
& I(a, 0, c) \\
& =\frac{1}{\left[\theta\left(1-\mathrm{e}^{-\lambda}\right) c-t_{2}\right]\left[\theta\left(1-\mathrm{e}^{-\lambda}\right)(a+c)-\left(t_{1}+t_{2}\right)\right]},
\end{aligned}
$$

where

$$
\begin{aligned}
& I(a, b, c) \\
& =\int_{0}^{\infty} \int_{x}^{\infty} \mathrm{e}^{t_{1} x+t_{2} y}[\bar{F}(x)]^{a}\left[h_{m}(F(y))-h_{m}(F(x))\right]^{b} \\
& \times[\bar{F}(y)]^{c} \mathrm{~d} y \mathrm{~d} x .
\end{aligned}
$$

Proof From (24), we have

$$
I(a, 0, c)=\int_{0}^{\infty} \mathrm{e}^{t_{1} x}[\bar{F}(x)]^{a} I(x) \mathrm{d} x,
$$

where

$$
I(x)=\int_{x}^{\infty} \mathrm{e}^{t_{2} y}[\bar{F}(y)]^{c} \mathrm{~d} y .
$$

On substituting for $\bar{F}(y)$ from (2) in (26), we get

$$
I(x)=\int_{x}^{\infty} \mathrm{e}^{-\left[\theta\left(1-\mathrm{e}^{-\lambda}\right) c-t_{2}\right] y} \mathrm{~d} y=\frac{\mathrm{e}^{-\left[\theta\left(1-\mathrm{e}^{-\lambda}\right) c-t_{2}\right] x}}{\left[\theta\left(1-\mathrm{e}^{-\lambda}\right) c-t_{2}\right]} .
$$

Upon substituting this expression for $I(x)$ in (25) and then integrating the resulting expression, we establish the result given in (23).

Lemma 3.2 For the distribution as given in (1) and any non-negative integers $a, b$ and $c$,

$$
\begin{aligned}
& I(a, b, c)=\frac{1}{(m+1)^{b}} \sum_{v=0}^{b}(-1)^{v}\left(\begin{array}{l}
b \\
v
\end{array}\right) \\
& \times \frac{1}{\left[\theta\left(1-\mathrm{e}^{-\lambda}\right)\{c+v(m+1)\}-t_{2}\right]} \\
& \times \frac{1}{\left[\theta\left(1-\mathrm{e}^{-\lambda}\right)\{a+c+b(m+1)\}-\left(t_{1}+t_{2}\right)\right]}, m \neq-1 \\
& =\frac{b !}{\left[\theta\left(1-\mathrm{e}^{-\lambda}\right)\right]^{2}\left[c-\left\{t_{2} / \theta\left(1-\mathrm{e}^{-\lambda}\right)\right\}\right]^{b+1}} \\
& \times \frac{1}{\left[a+c-\left\{\left(t_{1}+t_{2}\right) / \theta\left(1-\mathrm{e}^{-\lambda}\right)\right\}\right]}, \quad m=-1
\end{aligned}
$$

where $I(a, b, c)$ is as given in (24).

Proof Expanding $\left[h_{m}(F(y))-h_{m}(F(x))\right]^{b}$ binomially in (24) after noting that

$$
\begin{aligned}
& h_{m}(F(y))-h_{m}(F(x))=g_{m}(F(y))-g_{m}(F(x)) \text {, we get } \\
& I(a, b, c)=\frac{1}{(m+1)^{b}} \sum_{v=0}^{b}(-1)^{v}\left(\begin{array}{l}
b \\
v
\end{array}\right) \\
& \times \int_{0}^{\infty} \int_{x}^{\infty} \mathrm{e}^{t_{1} x+t_{2} y}[\bar{F}(x)]^{a+(b-v)(m+1)}[\bar{F}(y)]^{c+v(m+1)} \mathrm{d} y \mathrm{~d} x \\
& =\frac{1}{(m+1)^{b}} \sum_{v=0}^{b}(-1)^{v}\left(\begin{array}{l}
b \\
v
\end{array}\right) \\
& \times I(a+(b-v)(m+1), 0, c+v(m+1)) .
\end{aligned}
$$


Making use of Lemma 3.1, we establish the result given in (27).

When $m=-1, \quad I(a, b, c)=\frac{0}{0}$ as $\sum_{v=0}^{b}(-1)^{v}\left(\begin{array}{l}b \\ v\end{array}\right)=0$, so after applying L'Hospital rule and (15), (28) can be proved on the lines of (16).

Theorem 3.1 For Erlang-truncated exponential distribution as given in (1) and for

$$
\begin{aligned}
1 \leq r & <s \leq n, k=1,2, \cdots, m \neq-1, \\
& M_{X(r, n, m, k), X(s, n, m, k)}\left(t_{1}, t_{2}\right) \\
& =\frac{\left[\theta\left(1-\mathrm{e}^{-\lambda}\right)\right]^{2} C_{s-1}}{(r-1) !(s-r-1) !(m+1)^{r-1}} \\
& \times \sum_{u=0}^{r-1}(-1)^{u}\left(\begin{array}{c}
r-1 \\
u
\end{array}\right) I\left((u+1)(m+1), s-r-1, \gamma_{s}\right) \\
& =\frac{C_{s-1}}{(r-1) !(s-r-1) !(m+1)^{s-2}} \sum_{u=0}^{r-1} \sum_{v=0}^{s-r-1}(-1)^{u+v} \\
& \times\left(\begin{array}{c}
r-1 \\
u
\end{array}\right)\left(\begin{array}{c}
s-r-1 \\
v
\end{array}\right) \frac{1}{\left[\gamma_{s-v}-\left\{t_{2} / \theta\left(1-\mathrm{e}^{-\lambda}\right)\right\}\right]} \\
& \times \frac{1}{\left[\gamma_{s-v}-\left\{t_{2} / \theta\left(1-\mathrm{e}^{-\lambda}\right)\right\}\right]} .
\end{aligned}
$$

Proof From (5), we have

$$
\begin{aligned}
& M_{X(r, n, m, k), X(s, n, m, k)}\left(t_{1}, t_{2}\right)=\frac{\left[\theta\left(1-\mathrm{e}^{-\lambda}\right)\right]^{2} C_{s-1}}{(r-1) !(s-r-1) !} \\
& \times \int_{0}^{\infty} \int_{x}^{\infty} \mathrm{e}^{t_{1} x+t_{2} y}[\bar{F}(x)]^{m+1} g_{m}^{r-1}(F(x)) \\
& \times\left[h_{m}(F(y))-h_{m}(F(x))\right]^{s-r-1}[\bar{F}(y)]^{\gamma_{s}} \mathrm{~d} y \mathrm{~d} x
\end{aligned}
$$

upon using the relation (6). Now expanding $g_{m}^{r-1}(F(x))$ binomially in (31), we get

$$
\begin{aligned}
& M_{X(r, n, m, k), X(s, n, m, k)}\left(t_{1}, t_{2}\right) \\
& =\frac{\left[\theta\left(1-\mathrm{e}^{-\lambda}\right)\right]^{2} C_{s-1}}{(r-1) !(s-r-1) !(m+1)^{r-1}} \\
& \times \sum_{u=0}^{r-1}(-1)^{u}\left(\begin{array}{c}
r-1 \\
u
\end{array}\right) I\left((u+1)(m+1), s-r-1, \gamma_{s}\right) .
\end{aligned}
$$

Making use of Lemma 3.2, we establish the relation given in (30).

\section{Special Cases}

1) Putting $m=0, k=1$ in (30), the explicit formula for the joint moment generating function of order statistics of the Erlang-truncated exponential distribution can be obtained as

$$
\begin{aligned}
& M_{X_{r, s, n}}\left(t_{1}, t_{2}\right)=C_{r, s: n} \sum_{u=0}^{r-1} \sum_{v=0}^{s-r-1}(-1)^{u+v}\left(\begin{array}{c}
r-1 \\
u
\end{array}\right) \\
& \times\left(\begin{array}{c}
s-r-1 \\
v
\end{array}\right) \frac{1}{\left[n-s+1+v-\left\{t_{2} / \theta\left(1-\mathrm{e}^{-\lambda}\right)\right\}\right]} \\
& \times \frac{1}{\left[n-r+1+u-\left\{\left(t_{1}+t_{2}\right) / \theta\left(1-\mathrm{e}^{-\lambda}\right)\right\}\right]},
\end{aligned}
$$

where

$$
C_{r, s: n}=\frac{n !}{(r-1) !(s-r-1) !(n-s) !} .
$$

2) Putting $m=-1$ in (30), we deduce the explicit expression for joint moment generating function of upper $k$ record values for Erlang-truncated exponential distribution in view of (29) and (28) in the form

$$
\begin{aligned}
M_{\left(Y_{r}^{(k)}, Y_{s}^{(k)}\right)}\left(t_{1}, t_{2}\right) & =\frac{1}{\left[1-\left\{t_{2} / k \theta\left(1-\mathrm{e}^{-\lambda}\right)\right\}\right]^{s-r}} \\
& \times \frac{1}{\left[1-\left\{\left(t_{1}+t_{2}\right) / k \theta\left(1-\mathrm{e}^{-\lambda}\right)\right\}\right]^{r}} .
\end{aligned}
$$

Differentiating $M_{X(r, n, m, k), X(s, n, m, k)}\left(t_{1}, t_{2}\right)$ and evaluating at $t_{1}=t_{2}=0$, we get the product moments of gos when $m \neq-1$

$$
\begin{aligned}
& E[X(r, n, m, k) X(s, n, m, k)] \\
& =\frac{C_{s-1}}{(r-1) !(s-r-1) !} \\
& \times \frac{1}{\left[\theta\left(1-\mathrm{e}^{-\lambda}\right)\right]^{2}(m+1)^{s-2}} \sum_{u=0}^{r-1} \sum_{v=0}^{s-r-1}(-1)^{u+v} \\
& \times\left(\begin{array}{c}
r-1 \\
u
\end{array}\right)\left(\begin{array}{c}
s-r-1 \\
v
\end{array}\right) \frac{\gamma_{r-u}+2 \gamma_{s-v}}{\left(\gamma_{r-u}\right)^{3}\left(\gamma_{s-v}\right)^{2}}
\end{aligned}
$$

and when $m=-1$ that

$$
E\left[Y_{r}^{(k)} Y_{s}^{(k)}\right]=\frac{r(s+1)}{\left[k \theta\left(1-\mathrm{e}^{-\lambda}\right)\right]^{2}}
$$

and for $k=1$

$$
E\left[X_{U(r)} X_{U(s)}\right]=\frac{r(s+1)}{\left[\theta\left(1-\mathrm{e}^{-\lambda}\right)\right]^{2}} .
$$

Making use of (6), we can derive the recurrence relations for joint moment generating function of gos from (5).

Theorem 3.2 For the distribution given in (1) and for $1 \leq r<s \leq n, n \geq 2$ and $k=1,2, \cdots$ 


$$
\begin{aligned}
& \left(1-\frac{t_{2}}{\theta\left(1-\mathrm{e}^{-\lambda}\right) \gamma_{s}}\right) M_{X(r, n, m, k), X(s, n, m, k)}^{(i, j)}\left(t_{1}, t_{2}\right) \\
& =M_{X(r, n, m, k), X(s-1, n, m, k)}^{(i, j)}\left(t_{1}, t_{2}\right)+\frac{j}{\theta\left(1-\mathrm{e}^{-\lambda}\right) \gamma_{s}} \\
& \times M_{X(r, n, m, k), X(s, n, m, k)}^{(i, j-1)}\left(t_{1}, t_{2}\right) .
\end{aligned}
$$

Proof [10] has shown that for $1 \leq r<s \leq n, n \in N$, $m \in \mathfrak{R}, i, j=0,1,2, \cdots$ and a fixed positive integer $k \geq 1,1 \leq r \leq n, n \geq 2$,

$$
\begin{aligned}
& M_{X(r, n, m, k), X(s, n, m, k)}\left(t_{1}, t_{2}\right)=M_{X(r, n, m, k), X(s-1, n, m, k)}\left(t_{1}, t_{2}\right) \\
& +\frac{t_{2} C_{s-1}}{\gamma_{s}(r-1) !(s-r-1) !} \int_{0}^{\infty} \int_{x}^{\infty} \mathrm{e}^{t_{1} X+t_{2} y}[\bar{F}(x)]^{m} f(x) \\
& \times g_{m}^{r-1}(F(x))\left[h_{m}(F(y))-h_{m}(F(x))\right]^{s-r-1} \\
& \times[\bar{F}(y)]^{\gamma_{s}} \mathrm{~d} y \mathrm{~d} x .
\end{aligned}
$$

Differentiating both the sides of (34) $i$ times with respect to $t_{1}$ and then $j$ times with respect to $t_{2}$, we get

$$
\begin{aligned}
& M_{X(r, n, m, k), X(s, n, m, k)}^{(i, j)}\left(t_{1}, t_{2}\right)=M_{X(r, n, m, k), X(s-1, n, m, k)}^{(i, j)}\left(t_{1}, t_{2}\right) \\
& +\frac{t_{2}}{\theta\left(1-\mathrm{e}^{-\lambda}\right) \gamma_{s}} M_{X(r, n, m, k), X(s, n, m, k)}^{(i, j)}\left(t_{1}, t_{2}\right) \\
& +\frac{j}{\theta\left(1-\mathrm{e}^{-\lambda}\right) \gamma_{s}} M_{X(r, n, m, k), X(s, n, m, k)}^{(i, j-1)}\left(t_{1}, t_{2}\right),
\end{aligned}
$$

which, when rewritten gives the recurrence relation in (26).

At $t_{1}=t_{2}=0$ in (34), we obtain the recurrence relations for product moments of gos from Erlang-truncated exponential distribution in the form

$$
\begin{aligned}
& E\left[X^{i}(r, n, m, k) X^{j}(s, n, m, k)\right] \\
& =E\left[X^{i}(r, n, m, k) X^{j}(s-1, n, m, k)\right] \\
& +\frac{j}{\theta\left(1-\mathrm{e}^{-\lambda}\right) \gamma_{s}} E\left[X^{i}(r, n, m, k) X^{j-1}(s, n, m, k)\right] .
\end{aligned}
$$

One can also note that Theorem 2.1 can be deduced from Theorem 3.2 by letting $t_{1}$ tends to zero.

Remark 3.1 Putting $m=0, k=1$ in (34) and (35), we obtain the recurrence relations for joint moment generating function and product moments of order statistics for Erlang-truncated exponential distribution in the form

$$
\begin{aligned}
& \left(1-\frac{t_{2}}{\theta\left(1-\mathrm{e}^{-\lambda}\right)(n-s+1)}\right) M_{X_{r, s: n}}^{(i, j)}\left(t_{1}, t_{2}\right) \\
& =M_{X_{r, s-1: n}}^{(i, j)}\left(t_{1}, t_{2}\right)+\frac{j}{\theta\left(1-\mathrm{e}^{-\lambda}\right)(n-s+1)} M_{X_{r, s: n}}^{(i, j-1)}\left(t_{1}, t_{2}\right)
\end{aligned}
$$

and

$$
\begin{aligned}
E\left[X_{r, s: n}^{(i, j)}\right] & =E\left[X_{r, s-1: n}^{(i, j)}\right] \\
& +\frac{j}{\theta\left(1-\mathrm{e}^{-\lambda}\right)(n-s+1)} E\left[X_{r, s: n}^{(i, j-1)}\right]
\end{aligned}
$$

as obtained by [14] for exponential distribution at $i=j=1$ and $\theta\left(1-\mathrm{e}^{-\lambda}\right)=1$.

Remark 3.2 Substituting $m=-1$ and $k>1$, in (34) and (35), we get recurrence relations for joint moment generating function and product moments of upper $k$ record values for Erlang-truncated exponential distribution.

\section{Characterization}

Let $X(r, n, m, k), r=1,2, \cdots, n$ be $g o s$, then the conditional $p d f$ of $X(s, n, m, k)$ given $X(r, n, m, k)=x$, $1 \leq r<s \leq n$, in view of (4) and (5), is

$$
\begin{aligned}
& f_{X(s, n, m, k) \mid X(r, n, m, k)}(y \mid x) \\
& =\frac{C_{s-1}}{(s-r-1) ! C_{r-1}} \\
& \times \frac{\left[h_{m}(F(y))-h_{m}(F(x))\right]^{s-r-1}[\bar{F}(y)]^{\gamma_{s}-1}}{[\bar{F}(x)]^{\gamma_{r+1}}} f(y), \\
& x<y .
\end{aligned}
$$

Theorem 4.1 Suppose $F(x)<1$, for all $x \in(0, \infty)$ be a distribution function of the random variable $X$ and $F(0)=0, F(\infty)=1$, then

$$
\begin{aligned}
& E\left[\mathrm{e}^{t X(s, n, m, k)} \mid X(l, n, m, k)=x\right] \\
& =\mathrm{e}^{t x} \prod_{j=1}^{s-l}\left(\frac{\gamma_{l+j}}{\gamma_{l+j}-\left\{t / \theta\left(1-\mathrm{e}^{-\lambda}\right)\right\}}\right), l=r, r+1
\end{aligned}
$$

if and only if

$$
\bar{F}(x)=\mathrm{e}^{-\theta x\left(1-\mathrm{e}^{-\lambda}\right)}, x \geq 0, \theta, \lambda>0 .
$$

Proof From (36), we have

$$
\begin{aligned}
& E\left[\mathrm{e}^{t X(s, n, m, k)} \mid X(r, n, m, k)=x\right] \\
& =\frac{C_{s-1}}{(s-r-1) ! C_{r-1}(m+1)^{s-r-1}} \\
& \times \int_{x}^{\infty} \mathrm{e}^{t y}\left[1-\left(\frac{\bar{F}(y)}{\bar{F}(x)}\right)^{m+1}\right]^{s-r-1}\left(\frac{\bar{F}(y)}{\bar{F}(x)}\right)^{\gamma_{s}-1} \frac{f(y)}{\bar{F}(x)} \mathrm{d} y .
\end{aligned}
$$

By setting $u=\frac{\bar{F}(y)}{\bar{F}(x)}=\frac{\mathrm{e}^{-\theta y\left(1-\mathrm{e}^{-\lambda}\right)}}{\mathrm{e}^{-\theta x\left(1-\mathrm{e}^{-\lambda}\right)}}$ from (2) in (38) we obtain 


$$
\begin{aligned}
& E\left[\mathrm{e}^{t X(s, n, m, k)} \mid X(r, n, m, k)=X\right] \\
& =A \mathrm{e}^{t x} \int_{0}^{1} u^{\gamma_{s}-1-\left\{t / \theta\left(1-\mathrm{e}^{-\lambda}\right)\right\}}\left(1-u^{m+1}\right)^{s-r-1} \mathrm{~d} u
\end{aligned}
$$

where

$$
A=\frac{C_{s-1}}{(s-r-1) ! C_{r-1}(m+1)^{s-r-1}} .
$$

Again by setting $v=u^{m+1}$ in (39), we get

$$
\begin{aligned}
& E\left[\mathrm{e}^{t X(s, n, m, k)} \mid X(r, n, m, k)=x\right]
\end{aligned}
$$

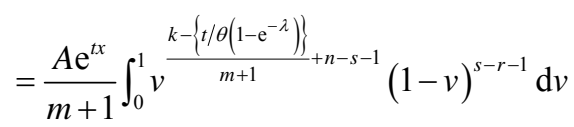

$$
\begin{aligned}
& =\frac{C_{s-1} \mathrm{e}^{t x}}{C_{r-1} \prod_{j=1}^{s-r}\left[\gamma_{r+j}-\left\{t / \theta\left(1-\mathrm{e}^{-\lambda}\right)\right\}\right]}
\end{aligned}
$$

and hence the relation given in (37).

To prove sufficient part, we have from (36) and (37)

$$
\begin{aligned}
& \frac{C_{s-1}}{(s-r-1) ! C_{r-1}(m+1)^{s-r-1}} \\
& \times \int_{x}^{\infty} \mathrm{e}^{t y}\left[(\bar{F}(x))^{m+1}-(\bar{F}(y))^{m+1}\right]^{s-r-1} \\
& \times[\bar{F}(y)]^{\gamma_{s}-1} f(y) \mathrm{d} y \\
& =[\bar{F}(x)]^{\gamma_{r+1}} H_{r}(x),
\end{aligned}
$$

where

$$
H_{r}(x)=\mathrm{e}^{t x} \prod_{j=1}^{s-r}\left(\frac{\gamma_{r+j}}{\gamma_{r+j}-\left\{t / \theta\left(1-\mathrm{e}^{-\lambda}\right)\right\}}\right) .
$$

Differentiating (40) both the sides with respect to $x$, we get

$$
\begin{aligned}
& -\frac{C_{s-1}[\bar{F}(x)]^{m} f(x)}{(s-r-2) ! C_{r-1}(m+1)^{s-r-2}} \\
& \times \int_{x}^{\infty} \mathrm{e}^{t y}\left[(\bar{F}(x))^{m+1}-(\bar{F}(y))^{m+1}\right]^{s-r-2} \\
& \times[\bar{F}(y)]^{\gamma_{s}-1} f(y) \mathrm{d} y \\
& =H_{r}^{\prime}(x)[\bar{F}(x)]^{\gamma_{r+1}}-\gamma_{r+1} H_{r}(x)[\bar{F}(x)]^{\gamma_{r+1}-1} f(x)
\end{aligned}
$$

or

$$
\begin{aligned}
& -\gamma_{r+1} H_{r+1}(x)[\bar{F}(x)]^{\gamma_{r+2}+m} f(x) \\
& =H_{r}^{\prime}(x)[\bar{F}(x)]^{\gamma_{r+1}}-\gamma_{r+1} H_{r}(x)[\bar{F}(x)]^{\gamma_{r+1}-1} f(x),
\end{aligned}
$$

where

$$
H_{r}^{\prime}(x)=t \mathrm{e}^{t x} \prod_{j=1}^{s-r}\left(\frac{\gamma_{r+j}}{\gamma_{r+j}-\left\{t / \theta\left(1-\mathrm{e}^{-\lambda}\right)\right\}}\right)
$$

and

$$
\begin{aligned}
& H_{r+1}(x)-H_{r}(x) \\
& =-\mathrm{e}^{t x} \prod_{j=1}^{s-r}\left(\frac{\gamma_{r+j}}{\gamma_{r+j}-\left\{t / \theta\left(1-\mathrm{e}^{-\lambda}\right)\right\}}\right) \frac{t / \theta\left(1-\mathrm{e}^{-\lambda}\right)}{\gamma_{r+1}} .
\end{aligned}
$$

Therefore,

$$
\frac{f(x)}{\bar{F}(x)}=-\frac{H_{r}^{\prime}(x)}{\gamma_{r+1}\left[H_{r+1}(x)-H_{r}(x)\right]}=\theta\left(1-\mathrm{e}^{-\lambda}\right)
$$

which proves that

$$
\bar{F}(x)=\mathrm{e}^{-\theta x\left(1-\mathrm{e}^{-\lambda}\right)}, x>0, \theta, \lambda>0 .
$$

\section{REFERENCES}

[1] A. R. El-Alosey, "Random Sum of New Type of Mixtures of Distributions," International Journal of Statistics and Systems, Vol. 2, No. 1, 2007, pp. 49-57.

[2] U. Kamps, "A Concept of Generalized Order Statistics," B. G. Teubner, Stuttgart, 1995.

[3] U. Kamps and E. Cramer, "On Distribution of Generalized Order Statistics,” Statistics, Vol. 35, No. 3, 2001, pp. 269-280.

[4] M. Ahsanullah and M. Z. Raqab, "Recurrence Relations for the Moment Generating Functions of Record Values from Pareto and Gumble Distributions," Stochastic Modelling and Applications, Vol. 2, No. 2, 1999, pp. 35-48.

[5] M. Z. Raqab and M. Ahsanullah, "Relations for Marginal and Joint Moment Generating Functions of Record Values from Power Function Distribution," Journal of Applied Statistical Sciences, Vol. 10, No. 1, 2000, pp. 27-36.

[6] M. Z. Raqab and M. Ahsanullah, "On Moment Generating Function of Records from Extreme Value Distribution," Pakistan Journal of Statistics, Vol. 19, No. 1, 2003, pp. 1-13.

[7] J. Saran and A. Pandey, "Recurrence Relations for Marginal and Joint Moment Generating Functions of Generalized Order Statistics from Power Function Distribution," Metron, Vol. LXI, No. 1, 2003, pp. 27-33.

[8] E. K. Al-Hussaini, A. A. Ahmad and M. A. Al-Kashif, "Recurrence Relations for Moment and Conditional Moment Generating Functions of Generalized Order Statistics," Metrika, Vol. 61, No. 2, 2005, pp. 199-220.

[9] E. K. Al-Hussaini, A. A. Ahmad and M. A. Al-Kashif, "Recurrence Relations for Joint Moment Generating Functions of Generalized Order Statistics Based on Mixed Population," Journal of Statistical Theory and Applications, Vol. 6, 2007, pp. 134-155.

[10] R. U. Khan, B. Zia and H. Athar, "On Moment Generating Function of Generalized Order Statistics from Gompertz Distribution and Its Characterization," Journal of 
Statistical Theory and Applications, Vol. 9, 2010, pp. 363-373.

[11] S. M. Ruiz, "An Algebraic Identity Leading to Wilson's Theorem," The Mathematical Gazette, Vol. 80, No. 489, 1996, pp. 579-582.

[12] Z. Grudzień and D. Szynal, "On the Expected Values of $k$-th Record Values and Associated Characterizations of Distributions," Proceedings of the 4th Pannonian Symposium on Mathematical Statistics, Reidel, 4-10 September
1983, pp. 119-127.

[13] M. Ahsanullah, "Record Statistics and the Exponential Distribution," Pakistan Journal of Statistics, Vol. 3A, 1987, pp. 17-40.

[14] N. Balakrishnan, H. J. Malik and S. E. Ahmed, "Recurrence Relations and Identities for Moments of Order Statistics," II: Specific Distributions, Communications in Statistics - Theory and Methods, Vol. 17, 1988, pp. 2657 2694. 